$\begin{array}{cl}\begin{array}{cl}\text { Revue } \\ \text { de Ihistoire } \\ \text { des religions }\end{array} & \text { Revue de l'histoire des religions } \\ & \begin{array}{c}1 \mid 2010 \\ \text { Beauté du rite }\end{array}\end{array}$

\title{
Beauté du chant, laideur du chantre : esthétique du plain-chant et dressage vocal au XVIII ${ }^{\mathrm{e}}$ siècle
}

The Beauty of the Song Vs. the Ugliness of the Singer: The Esthetics of Plainsong and Vocal Domestication in the 18th Century

\section{Xavier Bisaro}

\section{OpenEdition}

Journals

Édition électronique

URL : http://journals.openedition.org/rhr/7561

DOI : $10.4000 / \mathrm{rhr} .7561$

ISSN : 2105-2573

Éditeur

Armand Colin

Édition imprimée

Date de publication : 1 mars 2010

Pagination : 55-73

ISBN : 978-2200-92612-0

ISSN : 0035-1423

\section{Référence électronique}

Xavier Bisaro, « Beauté du chant, laideur du chantre : esthétique du plain-chant et dressage vocal au XVIII siècle ", Revue de l'histoire des religions [En ligne], 1 | 2010, mis en ligne le 01 mars 2013, consulté le 19 avril 2019. URL : http://journals.openedition.org/rhr/7561 ; DOI : 10.4000/rhr.7561 


\section{Beauté du chant, laideur du chantre : esthétique du plain-chant et dressage vocal au XVIII ${ }^{\mathrm{e}}$ siècle}

Marqueur du monde clérical mais massivement pratiqué par des laïcs, le plain-chant à l'époque moderne est un domaine partagé. Pour ses prescripteurs ecclésiastiques, la réflexion esthétique va de pair avec la définition des conditions d'une beauté vocale du chant ecclésiastique exposées par le biais de traités largement diffusés. Leur projet consiste également à cerner la réalité physique et sonore du chantre villageois dont la sauvagerie supposée est incompatible avec la conception que ces auteurs ont de la civilité musicale. Dresser les corps chantants indispensables à la concrétisation de leurs attentes pastorales et musicales : tel apparaît le défi auquel se confrontent les théoriciens du plain-chant au XVIII siècle.

\section{The Beauty of the Song Vs. the Ugliness of the Singer :}

\section{The Esthetics of Plainsong and Vocal Domestication} in the 18th Century

In the modern period, plainsong epitomized clerical society but was also largely practiced by lay people, and for this reason it is a shared field. The esthetical reflections of Church theorists on this subject lead them to define the conditions of vocal beauty in ecclesiastical singing and to expound them in widely distributed treatises. Their project consisted also of an effort to define the sonorous and physical reality of lay cantors, whose supposed savagery is not compatible with musical civility as conceived of by these authors. Thus, domesticating the singing bodies was essential to the fulfilment of their pastoral and musical expectations and appeared to be the main challenge of plainsong theorists in the 18th century. 
Le plain-chant dans la France d'Ancien Régime offre tous les paradoxes d'un domaine socialement partagé. Il est certes un marqueur d'appartenance au monde clérical en tant qu'élément de la discipline ecclésiastique; il suggère en outre une série d'actes techniques nécessitant une formation particulière en amont (maîtrise d'une notation, connaissance du latin, apprentissage de la psalmodie...). Mais le plain-chant déborde à plusieurs occasions de ce cadre. Dans ses formes processionnelles et rythmées (hymnes, séquences et proses), il est pratiqué par la plupart de la population; son appropriation est telle que ses mélodies sont fréquemment détournées pour des contrafacta spirituelles, politiques ou grivoises. Mais une autre échappée du plain-chant vers les laïcs se précise au cours du XVII ${ }^{e}$ siècle. Si les livres permettant le chant des principaux offices paroissiaux sont présents dans les chœurs d'églises rurales dès la fin $\mathrm{du} \mathrm{Xv}^{\mathrm{e}}$ siècle ${ }^{1}$, le chant lui-même est assuré le plus souvent par des officiers de chœur ecclésiastiques (tonsurés en attente d'ordination, vicaires, prêtres habitués). Or, les difficultés consécutives aux Guerres de Religion n'autorisent plus la présence d'un clergé en nombre suffisant dans les paroisses, ce qui compromet la continuité chantée du culte. Formés à partir de traités détaillant la messe pontificale - format liturgique surdimensionné pour leurs églises -, les curés sont parfois désemparés devant une telle situation : «faute d'Ecclésiastiques et Officiers, ils ne peuvent faire ce qu'ils voudraient bien $^{2} \gg$ pour solenniser dignement l'office divin. Par la force des choses, la codification des cérémonies inclura donc progressivement le cas de «l'Office moins solennel, où il y a peu d'Ecclésiastiques $^{3} »$. De plus, pour assurer le chant dialogué de la messe haute dominicale à laquelle s'ajoutent les vêpres, les offices mortuaires, les obits, et les matines certains jours de l'année, il faut désormais «dresser quelques laïques, gens d'âge et de probité au

1. $C f$. l'exemple des églises du diocèse de Rennes relevé par Bruno Restif, La Révolution des paroisses. Culture paroissiale et réforme catholique en HauteBretagne aux XVI et XVII siècles, Rennes, Presses universitaires de Rennes, 2006, p. 75.

2. Claude de La Croix, Le Parfaict Ecclésiastique, ou Diverses instructions sur toutes les fonctions cléricales, Paris, P. de Bresche, 1665.

3. Ibid., titre de la troisième partie. 
plain-chant ${ }^{4} \gg$. Sous la pression des circonstances, les portes du chœur doivent donc s'entrouvrir.

C'est au même moment que se précise dans les villages la figure du magister chantant ${ }^{5}$. À la faveur de la densification du maillage des petites écoles rurales, le maître d'école devient en effet l'auxiliaire naturel du curé. Sachant lire et écrire, ayant parfois reçu les ordres mineurs avant d'abandonner la voie du sacerdoce, le maitre détient, sous le regard du desservant, le droit d'encadrer la jeunesse du village, tout en devant lui enseigner le catéchisme, le chant et la lecture; il est enfin tenu d'assister le prêtre dans ses fonctions curiales. Dans les campagnes de l'époque moderne, le maître apparaît dès lors comme le point d'articulation entre l'exigence canonique du chant de la messe et son environnement prescriptif d'une part, et de l'autre l'énergie chantante des fidèles ${ }^{6}$. Durant les messes, son lieu de rattachement est à la frontière symbolique des deux mondes, la barrière du chœur : portant une chape (un ornement sacerdotal), il fait fonction de principal chantre posté devant son lutrin, mais autour duquel viennent d'agréger d'autres hommes de la communauté paroissiale. Par conséquent, le magister-chantre est un personnage indispensable et encombrant à la fois. Dans une société d'ordres, sa silhouette est étrangement floue : choisi par la communauté et le curé - sans que la priorité entre ces deux instances ne soit toujours claire ${ }^{7}-$, contractant avec la paroisse mais tributaire d'une approbation délivrée par l'autorité diocésaine, le

4. Extrait des instructions synodales d'Antoine Godeau, évêque de Grasse et Vence, cité par Monique Brulin, Le Verbe et la Voix. La manifestation vocale dans le culte en France au XVII siècle, Paris, Beauchesne, 1998, p. 320.

5. Au cours de cet article, les termes de maître, magister et régent désigneront une même fonction. Si les deux premières appellations sont reconnues par le Dictionnaire de l'Académie française pour désigner la personne chargée de l'enseignement scolaire paroissial, la dernière était normalement destinée au personnel enseignant de collège. Constatable notamment dans les archives paroissiales, son utilisation pour désigner les maîtres des petites écoles était néanmoins courante.

6. Le rôle du maître d'école-chantre comme intermédiaire est également observable dans le domaine administratif (le régent aidant à la rédaction d'actes ou à l'arpentage). Le profil socioprofessionnel de nombreux candidats à un contrat de maitre facilite d'ailleurs cette fonction de pivot; cf. Dominique Blanc, « Les saisonniers de l'écriture. Régents de villages en Languedoc au XVIII' siècle », Annales ESC, XLIII/4 (1988), p. 867-895.

7. La réalité de terrain est bien moins saisissable que ce que laisse entendre le Traité du gouvernement spirituel et temporel des paroisses de Jousse (Paris, Chez Debure, 1769, p. 11-12), ouvrage insistant sur le rôle du prêtre ou, éventuellement, des officiers du chœur dans la nomination des chantres. 
maître d'école est le produit d'une négociation. Sa voix est l'expression audible d'un malentendu, émanant d'une place mal définie qui ne sera que lentement investie par la littérature théorique et pédagogique consacrée au plain-chant.

\section{Actualisation d'Une Beauté ancestrale}

Dans un premier temps, cette voix semble étrangère à l'apologétique du plain-chant développée au cours du XVII siècle selon deux axes. Le premier consiste à valoriser la dimension intérieure du chant, à l'instar du processus de «mentalisation » que les théologiens font subir aux formes sensibles du culte. Expression extérieure de la foi, le plain-chant est ainsi promu par de nombreux auteurs au rang d'introducteur à l'oraison mentale. Symboliquement, sa pratique permet d'associer chaque individu au chœur angélique, et d'établir un rapport analogique entre l'Église de la terre et celle du ciel. Mais le chant ecclésiastique est aussi le support de communication de la Parole dans l'exercice du culte chrétien ${ }^{8}$. Influencés par l'ars bene pronuntiandi d'inspiration humaniste, les théoriciens post-tridentins considèrent que le chant ne saurait entraver la restitution du texte et, partant, sa capacité à imprégner l'intellect et l'âme des acteurs autant que des auditeurs de l'office'. Dans cette perspective, la validité réceptive - et non canonique - des actes rituels dépend de la rectitude d'énonciation des paroles qui les formulent, rectitude permise par une nécessaire implication formelle et spirituelle du locuteur chantant. Inspirés par Quintilien, les plain-chantistes de l'époque moderne intègrent en outre les gestes et les postures dans la définition de leur art du chant, art destiné à policer le verbe autant que le corps qui en résonne au moment de l'émettre.

Cette théologie du beau chant a pour conséquence la tentative de réguler la pratique des chantres, en essayant d'en contrôler la pro-

8. Brulin, op. cit., p. 4.

9. Jean-Jacques Duguet (Traité sur la prière publique et sur les dispositions pour offrir les ss. Mysteres, Paris, Jacques Estienne, 1707) dénonce ainsi le bruit confus de la psalmodie dans certaines églises au point que « le peuple ne saurait entendre ce que les chantres ne daignent pas écouter, quoiqu'ils le prononcent »; cité par Brulin, op. cit., p. 107. 
duction sonore pour la faire correspondre avec une nouvelle « éthologie du culte et des conduites du chant ${ }^{10} »$. La volonté d'encadrer les chantres n'est évidemment pas chose inédite tant elle appartient aux topiques de la littérature réglementaire ecclésiastique. Depuis les Pères jusqu'à saint Bernard, les traits d'une « voix congruente ${ }^{11}$ » réapparaissent régulièrement : prononciation distincte et non précipitée, virilité, gravité, modestie et dévotion fondent cet idéal vocal reformulé à chaque phase de l'histoire cyclique du réformisme ecclésiastique. Traditionnellement glosées d'un ouvrage à l'autre, réactivées par les critiques des humanistes - Érasme le premier s'attaquant aux débordements vocaux des chantres, ces autorités sont en plus d'un accès facilité grâce aux études patristiques et historiques produites par les érudits du XVII siècle. Elles fournissent par conséquent une vulgate sur mesure aux plain-chantistes, vulgate que, de surcroît, les volumes d'entretiens et de conférences ecclésiastiques et autres manuels des cérémonies de la messe transmettent massivement au personnel ecclésiastique paroissial. La norme vocale qui lui est proposée en exemple insiste ainsi sur la nécessité d'adopter une « voix grave, uniforme et distincte ${ }^{12}$ » sans cesse comprise entre les écueils du ni trop - ni trop peu. Appelé à se conformer à la dignité et à la décence imposées aux corps et, plus généralement, aux comportements ecclésiastiques, le plain-chant considéré comme un acte constitutif du culte divin participe donc pleinement du réformisme post-tridentin.

Lecteur assidu de saint Bernard, Guillaume-Gabriel Nivers (1632-1714) fournit dans sa Dissertation sur le chant grégorien un archétype de la transposition pour son siècle de l'exigence de mediocritas, de retenue et de savoir formulée par le cistercien. Après une apologie du plain-chant, Nivers expose une règle censée couper court à l'interventionnisme des chantres sur les mélodies notées : « Pour bien chanter le Pleinchant de l'Église, il n’y faut rien chanter, ajoûter, ou diminuer; mais simplement chanter

10. Jean-Yves Hameline, «Le plain-chant dans la pratique ecclésiastique aux lendemains du concile de Trente et des réformes postconciliaires», Plainchant et liturgie en France au XVII siècle, Paris/Versailles, Klincksieck/Éditions du Centre de musique baroque de Versailles, 1997, p. 28.

11. Brulin, op. cit., p. 263.

12. Manuel des Cérémonies romaines (1670) cité par Jean-Yves Hameline, "Célébrer "dévotement" après le Concile de Trente », La Maison-Dieu, n 218 (1999/2), p. 20 note 25. 
ce qui est dans le Livre $^{13}$ » afin de ne pas détruire «l'essence du Pleinchant, qui doit estre simple \& uny ${ }^{14} »$. Mais le chantre de paroisse rurale n'existe pas vraiment dans les préoccupations de ce musicien travaillant au service d'une des principales églises de Paris (Saint-Sulpice) et de Mme Maintenon - pour qui il fournit la musique de la Maison de Saint-Cyr. Nivers impute en effet les abus qu'il décrit aux diacres ou aux prêtres chantant l'évangile ou la préface, les « accens » et « langueurs » qu'ils ajoutent provenant de la musique mondaine porteuse de " nouveautez profanes ${ }^{15}$ ». Sa cible se découvre encore plus précisément au moment de stigmatiser « ceux qui imitent des Chants profanes du monde, pour chanter ou composer des Chants d'Église à la manière profane du siecle ». En somme, les déviances visées par Nivers concernent plutôt le chant des communautés nouvelles installées en milieu urbain - dont celui, très particulier, des oratoriens ${ }^{16}$. Il faut attendre la fin de son propos pour qu'apparaissent de nouveaux acteurs du chant ecclésiastique :

L'on peut encore attribuer ces voix profanes aux laïques, qui veulent chanter aux Offices Ecclesiastiques la Note dont ils ne scavent pas seulement la parole. Et c'est une chose déplorable d'entendre presque dans toutes les Eglises, les cacophonies, les incongruitez, les contre-temps, les faux Tons, \& toutes les confusions de chant que font ces gens là avec des manieres si ridicules, qu'ils choquent les sçavans, donnent de la peine aux autres, \& troublent le Service divin ${ }^{17}$.

L'attaque de Nivers reste pourtant vague dans sa destination. Elle peut être adressée autant aux paroisses rurales qu'urbaines; elle concerne par ailleurs un large spectre de laïques puisque Louis XIII, le prince de Condé et d'autres aristocrates affectionnaient de prendre part au chant de lutrin ${ }^{18}$. Lors des messes auxquelles ils assistaient, ils devenaient alors susceptibles de mêler chant ecclésiastique et style ornemental de l'air de cour qu'ils pratiquaient également.

13. Guillaume-Gabriel Nivers, Dissertation sur le chant grégorien, Paris, Aux dépens de l'Autheur, 1683, p. 51.

14. Ibid., p. 52. Nivers n'exclut néanmoins pas «quelques ports de voix » ainsi que « quelques diêses [...] que l'on fait mesme sans y penser»; ibid., p. 53.

15. Ibid., p. 53.

16. Philippe Vendrix, « Pour les grands et les autres : la réforme oratorienne du plain-chant », Plain-chant et liturgie..., p. 87-96.

17. Nivers, op. cit., p. 57.

18. Pour l'exemple d'Henri II de Condé, $c f$. le témoignage rapporté par Abraham-Nicolas Amelot de La Houssaie, Memoires historiques, politiques, critiques et litteraires, Amsterdam, Chez Zacharie Chatelain, 1737, t. II, p. 408-409. 
La réalité du terrain paroissial extra-urbain est en revanche plus perceptible dans les ouvrages normatifs destinés aux prêtres et, plus rarement, aux fidèles. En matière de chant, la préoccupation principale de ces textes consiste à répartir dans l'église les laïcs susceptibles d'y prendre part. Or, même si le chantre-maître d'école bénéficie de plein droit d'un accès au chœur, le recours à d'autres hommes adultes vient brouiller la répartition binaire chœur-nef, situation que le cérémonial parisien de 1658 tente de circonscrire :

Les hommes et garçons doivent être aux côtés de l'église à l'entour du chœur, et les femmes et les filles dedans, au côté de la nef... les hommes qui peuvent chanter le font bien plus commodément étant plus près du chœur et les femmes qui ne doivent pas chanter, prient plus attentivement : ce qui est même plus dans l'honnêteté et dans la bienséance chrétienne.

On peut toutefois donner place dans le chœur aux hommes et aux garçons qui savent le plain-chant et aux jeunes enfants, qui peuvent servir aux cérémonies de l'autel et du chœur, ès églises où on en a besoin et pour cette nécessité seulement ${ }^{19}$.

Le pragmatisme pacifique de ces textes à visée organisatrice plus que réformatrice n'est perturbé qu'à de rares occasions. Paradoxalement, c'est du général oratorien Bourgoing, commanditaire du « nouveau » plain-chant déploré par Nivers, que vient un de ces coups de semonce :

Il y a [dans les paroisses rurales] si peu d'ecclésiastiques que les curés sont contraints d'emprunter le secours d'aucuns de leurs paroissiens qui, la plupart, sont sans capacité et bien souvent sans piété, donnant plutôt scandale que dévotion, soit par la dissonance de leur chant, soit aussi par leur mauvaise posture et incomposition du corps ${ }^{20}$.

L'accusation d'ignorance est bien là, mais doublée par de nouveaux attendus. L'impiété est désormais associée à la défaillance technique (le chant « dissonant»), alors qu'une image vient sceller les termes du portrait, celle de corps « incomposés » dont la seule

19. Cité par Brulin, op. cit., p. 322. Cf. la même proposition faite dans le Livre d'Église à l'usage des laics du diocèse d'Amiens, Amiens, Chez Charles Caron Hubault, 1704.

20. Extrait du David françois ou Traité de la saincte psalmodie du père Bourgoing (1641), cité par Brulin, op. cit., p. 378-379. Autre critique des « laïques et des paysans revêtus de chapes [qui] chantent les saint Offices » chez Tronson, Examens particuliers sur divers sujets propres aux ecclésiastiques et à toutes les personnes qui veulent s'avancer dans la perfection, Nantes-Marseille, Chez $\mathrm{M}^{\mathrm{me}}$ la

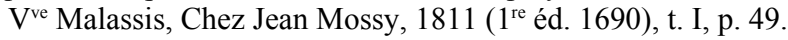


vue est cause de scandale. À la suite de Bourgoing, cette laideur vocale, physique et morale des chantres de village n'allait pas tarder à devenir un thème récurrent des traités de chant ecclésiastique au XVIII ${ }^{\mathrm{e}}$ siècle.

\section{DES TRAITÉS DE REDRESSEMENT}

Les ouvrages abordant la question sont surtout le fait de clercs de rang intermédiaire (éventuellement curés de paroisse comme les deux abbés Poisson ${ }^{21}$ ) ou de musiciens au service de chapitres cathédraux $^{22}$. Abordant la pratique du plain-chant, leurs objectifs ne sont pas forcément identiques ${ }^{23}$, pas plus que l'ampleur de leur diffusion. Si certains de ces traités résultent d'une réforme de liturgie diocésaine dont ils accompagnent l'apprentissage du nouveau chant (Lebeuf à Paris, Hardouin à Reims, Oudoux à Noyon), d'autres ont une portée plus étendue. Couplé à un graduel portatif, le traité de La Feillée connaîtra ainsi une diffusion extensive jusque tard dans le XIX ${ }^{\mathrm{e}}$ siècle. Le principal point commun de ces livres réside plutôt dans leur lectorat potentiel. Hormis l'énigmatique publication du frère Carré ${ }^{24}$, ils sont destinés en premier aux personnes « chargées de gouverner l'Office divin » (Oudoux), aux « jeunes ecclésiastiques » (La Feillée) ou aux «Séminaires \& aux Maîtres de Chantres » (L. Poisson), autrement dit à ceux qui encadrent les chantres, ces derniers n'apparaissant que rarement dans les pages de titre et les préfaces. Le clergé de second ordre trouve donc dans

21. Chanoine de la cathédrale d'Auxerre avant de devenir académicien, l'abbé Jean Lebeuf est le seul à déroger à ce profil.

22. Les traités abordés dans le cadre de cet article sont référencés en annexe. Pour une liste complète des traités publiés au cours des $\mathrm{XVII}^{\mathrm{e}}$ et $\mathrm{XVIII}{ }^{\mathrm{e}}$ siècles, cf. Bénédicte Mariolle, «Bibliographie des ouvrages théoriques traitant du plainchant (1582-1789) », Plain-chant et liturgie..., p. 285-356.

23. Une typologie sommaire distinguerait les traités purement pratiques (La Feillée, Oudoux, Hardouin, Poisson) de ceux consacrés à l'esthétique et à la composition du chant (L. Poisson, Cousin), ou encore ceux mêlant érudition historique et pédagogie pratique (Lebeuf).

24. Bien que sa destination monastique soit clairement exprimée, ce traité est de format anormalement grand, et ses informations concernant le chant alternent avec de nombreuses pages consacrées aux remèdes contre le rhume ou à l'éloge d'une consommation raisonnée du vin! L'ouvrage est, de plus, ignoré par les commentateurs du XVIII ${ }^{\mathrm{e}}$ siècle. 
ces publications homogènes ${ }^{25}$ de quoi adopter lui-même un idéal de chant, de quoi le guider au moment d'agréer un régent d'école, voire de quoi orienter la formation plain-chantesque des fidèles masculins de la paroisse.

Cette littérature définit tout d'abord les conditions de l'aptitude physique et comportementale au chant. Pour tous les auteurs, la «bonne » voix est une propriété innée, et son antithèse - la voix « rude »- est à éloigner du lutrin, ou à corriger par le travail. L'intrication du lutrin et de la sociabilité masculine villageoise (avec ses inimitiés, ses préséances et ses cooptations) est ainsi ignorée au profit d'une norme vocale que viennent encore renforcer les codes d'une civilité particulière :

Celui qui a la Voix trop forte en comparaison des autres, doit la donner à proportion de la grandeur de l'Église où il chante; car sans cela il fatigueroit les autres, qui pour se faire entendre forceroient trop leur Voix; \& s'il ne sçavoit pas le Chant, ou qu'il n'en fût pas sûr, il troubleroit le Chœur : en ce cas il faut qu'il dirige sa Voix d'une façon qu'il puisse entendre les autres, pour se regler. S'il ne sçait rien du tout, ou qu'il ne chante que par routine, il doit encore plus s'appliquer à écouter les autres; s'il a la Voix tout à fait discordante, il ne doit jamais chanter que les choses auxquelles il est obligé par son état ${ }^{26}$.

Le chantre doit donc se fondre dans un ensemble en fonction de la puissance de sa voix et de sa capacité à lire la notation musicale : pour ces auteurs, la seule volonté de s'approcher du lutrin et la débrouillardise du débutant ne suffisent pas. En cela éloigné de la réalité des lutrins de paroisses, leur plain-chant est également rêvé en termes techniques. Une fois son corps stabilisé et sa puissance vocale correctement dosée, le chantre idéal devrait effectivement maîtriser l'émission du texte qu'il chante :

Il faut éviter un défaut essentiel dans le Chant, qui est de ne point aspirer les Notes dans leurs liaisons, en donnant des coups de Voix épouvantables, $h a, h a$, he, he, sous pretexte que le Plain-chant doit être plain, selon son étymologie, \& battu; l'on peut fort bien le battre $\&$ le rendre plain, soit dans les liaisons des Notes, soit sur les mots, en

25. Les emprunts d'un traité à l'autre sont très fréquents. Ainsi, la section consacrée au plain-chant chez Imbert est, mot pour mot, identique à celle de Carré. Oudoux reconnaît pour sa part avoir cherché « à recueillir \& à réunir ce qu'on ne trouveroit qu'avec des recherches fatigantes dans un grand nombre de Traités sur le Chant, que tout le monde n'est point en état de se procurer, \& que peu sont capables de bien saisir »; Oudoux, p. 3.

26. La Feillée, p. 68. 
appuyant dessus sans affecter de donner des coups de Voix trop forts, en ouvrant \& refermant la bouche à chaque coup de Voix que l'on donne à chaque Note, $\&$ en prononçant les voyelles qui gouvernent le mot que l'on chante, \& qui servent aux liaisons; comme s'il y avoit une $H$ aspirée à chaque fois qu'on les réitère, ou souvent sans faire d'aspiration, par un mouvement des lèvres. Il y en a qui articulent toutes ces Notes liées comme s'il y avoit ces deux voyelles üé ou $\ddot{u} i$; il faut que la bouche reste ouverte de la façon naturelle qu'elle doit être pour chaque voyelle sur laquelle on doit faire les liaisons, laissant sortir sa Voix naturelle \& sans contrainte ${ }^{27}$.

Changeant d'échelle, la mise en conformité passe du corps aux mouvements des mâchoires, des lèvres et de l'ensemble du visage, ainsi qu'aux coups de gorge ${ }^{28}$. Le style vocal défini par ces traités se précise alors : il implique un chant lié - et donc contraire à la vocalité très articulée des chantres -, et respectueux des phonèmes constitutifs du texte latin. En somme, selon l'abbé Lebeuf, un chant produit par des voix « qui éxécutent avec grace ce qu'il y a de doux $\&$ de mélodieux dans l'arrangement des sons $^{29} »$.

La coordination des syllabes des textes chantés en dépit des respirations imposées par la lenteur propre au plain-chant constitue l'ultime domaine d'intervention des théoriciens. En lutte contre les respirations dictées par le découpage graphique de la mélodie notée (figure $1 a)$, La Feillée suggère un système de respiration excluant toute séparation de deux syllabes appartenant à un même mot (figure 1b).

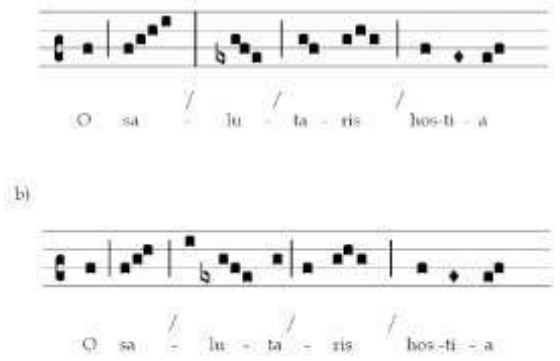

Figure $1 a$ et $1 b$ - Respirations de chantre corrigées par La Feillée

27. La Feillée, p. 67-68.

28. «Il faut prononcer naturellement, pourvû qu'on le fasse bien, sans grimace, sans contorsion de la bouche, \& sans trop d'affectation, \& autant que l'on peut sans remuer les lévres dans les liaisons du Chant, parce que cela fait varier l'articulation »; La Feillée, p. 77. Conseils identiques chez Carré, p. 36, et Poisson, p. 402-403.

29. Lebeuf, p. 106. 
Un régent d'école et les chantres laïcs adoptant les consignes proposées par ces auteurs doivent donc modérer, surveiller, égaliser, corriger, sans oublier l'effort de compréhension et de mémorisation que représente l'assimilation de l'attirail théorique du plain-chant (modes, terminaisons psalmodiques...). En définitive, le naturel du chant prôné par les traités s'oppose à un autre naturel, celui du chantre de paroisse. Arrivant au lutrin avec la dynamique corporelle de la " vraie vie », avec l'expérience d'un peu-lisant et un rapport au chant marqué par les ressorts de la sociabilité villageoise, le chantre ne peut ressembler à l'image véhiculée par ces traités qu'à condition d'abandonner son être. Pressentant la difficulté, ces traités insistent donc sur la formation des écoliers au chant, ce qui conduit Oudoux à présenter sa méthode à la manière d'un catéchisme dialogué. Proposée par tous les auteurs, la décomposition de l'apprentissage en phases intangibles (solfier le nom des notes, chanter avec le nom des notes, chanter avec le texte) cherche pareillement à rationaliser un encadrement et vise à exclure tout écart; pour ce faire : «Il faut défendre à un Ecolier d'étudier seul jusqu'à ce qu'il en soit capable, cela seroit dangereux pour lui, parce qu'il prendroit des tons mauvais qui lui resteroient $t^{30}$.» Les exercices systématisés par ces ouvrages prennent d'ailleurs l'apparence d'une épellation musicale proche des techniques d'apprentissage de la lecture pratiquées par les régents de petites écoles, et dont la répétition est censée imprimer la matière malléable de l'intelligence enfantine (figure 2).

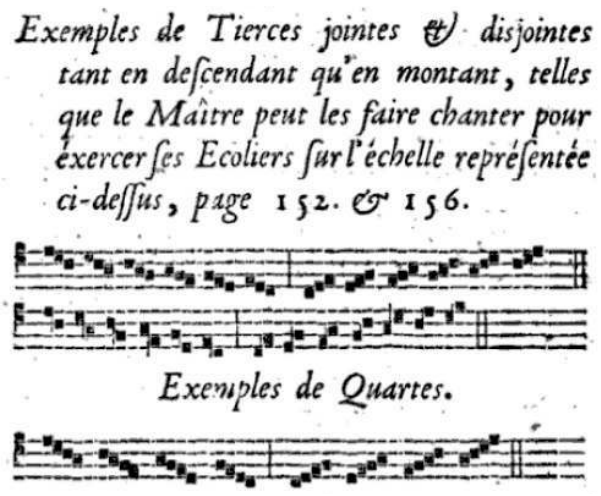

Figure 2 - Traité pratique sur le chant ecclésiastique de Lebeuf (p. 289)

30. La Feillée, p. 87. 
Toutefois, en dehors d'une insistance sur la restitution correcte du latin chanté, un tel contenu technique et pédagogique reste proche des prescriptions vocales et cérémonielles enseignées aux membres du clergé ${ }^{31}$. À la marge des consignes principales se façonne pourtant la connotation sociale du chant que cette littérature cherche à circonvenir. Les conseils prodigués par Oudoux cassent ainsi les rapports horizontaux développés à partir du lutrin paroissial. Suggérant de donner les chantres « sur-tout de la Campagne $^{32} \gg$ en contre-exemple aux écoliers, il brise l'élan imitatif des enfants à l'écoute des adultes; signalant que l'auteur d'excès vocaux est « le plus ignorant», il relègue ces conduites déviantes dans le domaine « des cris affreux $\&$ des sons dénaturés, qu'on prendroit pour des hurlemens $\&$ des cris poussés à dessein ${ }^{33} »$. Alors que le plain-chant doit essentiellement « faire sentir l'énergie des paroles ${ }^{34} »$, son détournement sémantique au travers des pratiques de lutrin pousse ces auteurs lettrés à reléguer la voix des chantres dans le domaine du bruit : symboliquement, Rousseau utilise le chant des paroisses comme illustration du verbe « brailler » de son Dictionnaire de musique ${ }^{35}$. Au même moment, le maître d'école, figure centrale de ce chant in-concevable, devient le paria des débats que l'enseignement primaire nourrit durant les années 1760. Même pour certains prêtres, le magister n'est qu'un parvenu ecclésiastique et intellectuel :

C'est un jeune homme qui sort de l'école, qui craint le sort de la milice, qui a été enfant de chœur dans son village, qui sait lire jusqu'à trouver l'office du jour dans un livre d'église, qui chante au lutrin, qui écrit, quoique machinalement et sans principes, qui sait tant bien que mal les premières règles de l'arithmétique... il se présente, il fait éclater une voix qui approche de celle de Stentor; on le croit habile; il

31. $C f$. par exemple le chapitre «Du chant du Chœur» du Cérémonial du diocèse de Langres, Langres, Chez Pierre Defay, 1775.

32. Oudoux, p. LXVIJ.

33. Ibid., p. LXVIIJ.

34. L. Poisson, p. 93. En dépit de son opposition à Poisson, propos identiques chez Cousin de Contamine, Traité critique du Plain-chant, Paris, Chez P.G. Le Mercier, 1749 , p. 55

35. «Brailler : c'est excéder le volume de sa voix, chanter tant qu'on a de force, comme font au lutrin les Marguilliers de Village, \& certains Musiciens ailleurs »; Jean-Jacques Rousseau, Dictionnaire de musique, Paris, Chez la Veuve Duchesne, 1768, p. 58. 
est reçu avec applaudissement. M. le Magister se pavane dans l'église avec une chape sur le corps, et peu s'en faut qu'il ne veuille faire la loi au curé de la paroisse ${ }^{36}$.

\section{UN CHANT INCOMPRÉHENSIBLE}

L'opposition à cette dépréciation est difficilement décelable. De rares chantres investissent le monde de l'écrit public pour instiller de la complexité dans le tableau trop simple de leur supposée décrépitude. Rappelant son degré de connaissances dans plusieurs domaines du savoir, un régent affirme dans un journal « [posséder] la méthode du plain-chant sans avoir une voix de $\operatorname{Stentor}^{37} \gg$ et, par conséquent, pouvoir l'enseigner par principes. Le simple fait qu'il formule sa protestation par voie de presse atteste son intégration au lectorat éclairé de la fin du XVIII ${ }^{\mathrm{e}}$ siècle. Des témoignages indirects confirment pareillement que le régent de village ne peut se laisser réduire au seul profil de l'ignorant orgueilleux. Le diariste Pierre Prion émet par exemple un tout autre avis sur le régent-chantre de la paroisse d'Aubais :

[...] il était très savant, possédant pour le moins aussi bien le latin que Cicéron. Il avait encore tant d'autres riches talents. Son écriture surpassait celle faite au burin. L'algèbre, l'arithmétique [sic]. C'était, après, un chantre qui par sa voix faisait tous les jours retentir nos églises. L'on peut dire homme de bonnes mœurs, bonne conversation, sobre, gracieux et bienfaisant ${ }^{38}$.

Mais la plus frappante des résistances de la culture cantorale à sa caricature s'observe en creux, dans la persistance des consignes adressées par les théoriciens. Constante durant deux siècles, leur velléité correctrice est manifestement entrée en conflit avec des habitudes tenaces contredisant les données de l'actio canendi régulatrice qu'ils tentent d'instaurer ${ }^{39}$. Parmi les caractéristiques de l'immutabilité des chantres, leur tonitruance est la moins comprise, certai-

36. Courtalon (curé), « Projet en faveur des petites écoles des campagnes », Journal de Troyes (1784), cité par Albert Babeau, L'école de village pendant la Révolution, Paris, Librairie académique Didier - Émile Perrin, 1885 ( $2^{\mathrm{e}}$ éd.), p. 20.

37. Ibid., p. 21.

38. Cité par Blanc, art. cit., p. 889.

39. Hameline, art. cit., p. 22. 
nement en raison d'une portée anthropologique à la fois invisible (car dissimulée dans les replis de la société rurale) et inavouable. En effet, quelle que soit la place du chantre au sein de la communauté paroissiale, sa voix entre dans une compétition de puissance engageant la crédibilité de sa représentation à l'oreille des fidèles. Ainsi que Bernard Lortat-Jacob l'a analysé à propos des chantres de confréries sardes, " chanter est d'abord un défi ( $s$ fida) : c'est se faire entendre des autres et, plus encore, chercher à dominer de sa propre voix les compagnons du chœur - ce qui invite ces derniers (et même les oblige) à chanter également fort, quitte à se briser la voix ${ }^{40} \gg$. À cette concurrence intra-paroissiale s'ajoute celle existant entre villages voisins : à côté des coqs de villages et d'autres indices de prestige communautaire figure la force vocale du lutrin ${ }^{41}$. Le statut individuel de certains chantres en accentue l'importance. Lorsque les marguilliers se regroupent devant le lutrin, l'adhésion collective dont ils bénéficient doit être vérifiée par leur capacité à tenir leur rang vocalement. Quant au régent d'école, sa puissance vocale est encore plus décisive. Ce critère étant pris en compte au moment de son choix par le village et le curé ${ }^{42}$, il fonde sa légitimité le temps de son engagement qui est souvent saisonnier. L'espoir d'être reconduit, le résultat de ses éventuelles quêtes, mais aussi le simple respect de son contrat l'entraînent à ne pas démériter dans l'escalade sonore résultant de la dynamique de lutrin.

La déstructuration du texte au moment de l'acte de chant est un autre constat dont la récurrence dans les descriptions atteste la prégnance des habitudes de lutrin. Forcément ignorants, les chantres seraient ainsi à l'origine de la perte de sens des textes latins chantés auprès des fidèles. Outre une conception pour le moins optimiste du lien entre le latin et le plus grand nombre des fidèles (pour qui le français parisien est déjà une réalité lointaine), cette appréciation priorise la portée sémantique du mot, là où le plain-chant paroissial

40. Bernard Lortat-Jacob, «Prononcer en chantant. Analyse musical d'un texte parlé (Castelsardo, Sardaigne) », L'Homme, XXXVIII (1998), p. 110.

41. En 1764, un curé du diocèse de Saint-Papoul se félicite de son chantre principal : « tous les étrangers sont satisfaits de son chant, il est [sic] pas village où l'on chante mieux »; cité par Blanc, art. cit., p. 883.

42. $C f$. le cas du chantre analphabète mais bon chanteur choisi par les villageois et le curé de Quiers, cité par Jacques Houdaille, « Les signatures au mariage de 1740 à $1829 »$, Population, XXXII/1 (1977), p. 71 note 2. 
est en avant tout affaire de « surface phonétique ${ }^{43}$ ». Le magister et les hommes qui se joignent à lui sont en effet les producteurs d'un son dont les caractéristiques sont d'abord calendaires (le dimanche, les fêtes, les sépultures), spatiales (le chœur paroissial et ses abords), assonantes (les phonèmes récurrents du latin), rythmiques (l'allégement des syllabes non accentuées dans un débit général plutôt lent) et timbriques (des chantres masculins «poussant» leur voix). Ce chant n'a donc pas forcément vocation à être compris; il doit surtout être efficient, prendre sa part au rituel afin d'agir pour le bien de la communauté dont il est une émanation. Surpersonnalisé par ses acteurs - les marguilliers, le régent, un voisin... - ce chant est dépersonnalisé par son enveloppe sonore, identique et collective quelle que soit la composition du groupe chantant. Il est un masque qu'endossent les hommes du lutrin, le masque de la coutume, peutêtre même celui des ancêtres ${ }^{44}$. L'entêtement du discours théorique à lutter contre les signes d'existence et de légitimité de ce chant irréductible doit donc engager à dialectiser sa fonction : plutôt que médiateur, il est frontière (en tant que séparation, point de contact par juxtaposition et potentielle zone d'échange) entre les attentes irréconciliables de ses auteurs et de ses récepteurs.

\section{UNE VOIX EN SURSIS}

Le chant du magister à la fin de l'Ancien Régime est solidement imbriqué dans la communauté paroissiale : par contrat, le régent ne s'engage-t-il pas à officier les dimanches et fêtes, mais aussi « tous les jours où les habitants demanderont ${ }^{45}$ »? Il est pourtant en phase de fragilisation. À l'époque de l'envahissement du propos esthétique par le sentiment, le décalage sonore que plain-

43. Jacques Cheyronnaud, Le Lutrin d'église et ses chantres au village (XIX - XXe siècles). Approche d'un service public musical, thèse de l'École des hautes études en sciences sociales, 1984, p. 242.

44. L'assimilation du chant de masque rituel au chant des ancêtres est bien connue des ethnomusicologues; $c f$. Marie-Paule Ferry, « Voix des masques, voix des esprits », Pour une anthropologie de la voix, Paris, L'Harmattan, 1993, p. 329339. En tenant compte des multiples interactions entre religion institutionnelle et pensée magique rurale, l'attachement des populations au chant de lutrin mériterait d'être envisagé sous cet angle.

45. Clause de contrat d'engagement du maitre d'école d'une paroisse de Franche-Comté au XvIII ${ }^{e}$ siècle, citée par Cheyronnaud, op. cit., p. 469. 
chant de lutrin instaure aggrave sa marginalité. Les flux et reflux du goût musical urbain attaquent la stylistique du chant ecclésiastique - ce dont témoigne la controverse en 1749-50 entre Cousin de Contamine, partisan d'un rapprochement de la musique et du plain-chant et le chanoine Roulleau, défenseur du maintien du plain-chant en l'état ${ }^{46}$-, et annule progressivement la recharge de respectabilité dont ce dernier bénéficia au XVII ${ }^{\mathrm{e}}$ siècle grâce au rayonnement de congrégations nouvelles ou réformées et des répertoires qui leur étaient associés. Le plain-chant est désormais l'attribut sonore d'un monde dont les figures chantantes et, plus généralement, les institutions encadrantes sont moins bien comprises qu'auparavant ${ }^{47}$.

Les physiocrates et d'autres philanthropes intéressés par le sort des populations rurales s'approchent également des acteurs du chant ecclésiastique. Les tenants d'une réforme utilitariste de l'enseignement scolaire ${ }^{48}$ étant favorables à l'introduction des mathématiques, de l'arpentage et du dessin, l'école du village leur apparaît comme un lieu figé dans le formalisme, un lieu gêné par sa tutelle ecclésiastique et hostile aux transformations du temps ${ }^{49}$, un lieu d'où provient une langue - latin ânonné de l'école, latin hurlé du lutrin - dissimulant des sens cachés, des usages que l'on devine insaisissables. Avant que la Convention révolutionnaire ne cherche à remodeler l'expression orale des ruraux ${ }^{50}$, le code de communication dont

46. Cousin de Contamine, Traité critique du Plain-chant, Paris, Chez P.G. Le Mercier, 1749; chanoine Roulleau, « Defense du Chant Gregorien », Mercure de France, mai 1750, p. 41-77. Rousseau évoquera lui aussi la question du statut esthétique du plain-chant; Rousseau, op. cit., p. 373-378.

47. Jean-Louis Jam, "Images du chantre au XVIII ${ }^{\mathrm{e}}$ siècle ", Maîtrises et Chapelles aux XVII \& XVIII siècles, Clermont-Ferrand, Presses universitaires BlaisePascal, 2003, p. 409-425. Ce discrédit touche plus généralement les principales institutions ecclésiastiques non paroissiales (chapitres séculiers, ordres religieux) ainsi que le chant qui leur est propre; $c f$. Philippe Bourdin, "Collégiales et chapitres cathédraux au crible de l'opinion et de la Révolution ", Annales historiques de la Révolution française, $\mathrm{n}^{\circ} 331$ (2003), p. 29-54.

48. Harvey Chisick, "Institutional innovation in popular education in eighteenth century France: two examples », French Historical Studies, X/1 (1977), p. 41-73.

49. Mireille Laget, "Petites écoles en Languedoc au XVIII ${ }^{\mathrm{e}}$ siècle », Annales, XXVI/6 (1971), p. 1411. Cf. la description satirique des petites écoles chez Sébastien Mercier, Tableau de Paris, Amsterdam, s. n., 1782, t.III, chapitre XLIII.

50. Michel de Certeau, Dominique Julia, Jacques Revel, Une politique de la

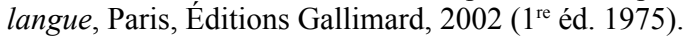


le magister est garant est déjà contesté ${ }^{51}$. Enfin, son lutrin est un endroit trop enclin à provoquer des « conduites effervescentes ${ }^{52}$ » alors qu'il devrait idéalement symboliser le point de rencontre entre modèles institutionnels et conduites laïques. En situation de conflit entre communauté villageoise et prêtre desservant, les chantres connaissent en effet le caractère primordial de leur voix tant pour les fidèles que pour le ministre du culte. Leur chant autant que leur silence peut alors compromettre la régularité de la vie paroissiale, comme à Fitou en 1784 :

Le jour de Saint-Pierre, M. le Curé allant faire la procession qu'il est d'usage de faire avant la messe, et étant au pied de l'autel, entonna l'himne des apôtres, personne ne répondit, il se fit un murmure dans l'église, le curé se tourna et vit le second consul et ledit Fabre qui rioient de son embarras. M. le Curé fut obligé de dire une messe basse, ceux qui ont fait le complot de ne point chanter, empêchoient de chanter ceux qui en avoient envie en leur disant, il faut laisser égoziller M. le Curé, puisqu'il a donné au maître d'école un certificat en latin contre lui ${ }^{33}$.

L'incident pourrait faire sourire; il concerne pourtant la messe chantée paroissiale, office que le curé doit canoniquement faire chanter aux dimanches et fêtes pour ses paroissiens et en leur nom, mais qui est en outre indispensable à la vie rurale d'Ancien Régime ${ }^{54}$. Son interruption provoque ici le murmure, manifestation courante de l'incrédulité mais aussi de la menace sociale. Il touche à la figure centrale du magister dont le statut est défendu au moyen d'un de ses attributs, i.e. le droit de sonoriser le rite. Le plain-chant provoque de surcroît une coalition contre nature : les consuls et le maître-normalement au service du curé - puis, de gré ou de force, ceux qui, hors du complot, voulaient chanter. Outil de contrôle et manifestation d'autorité sur les populations, la messe paroissiale est finalement détournée de sa vocation. Fédérateur tout en suscitant contestation

51. Cf. par exemple les perfidies de La Feuille villageoise, III (1792), p. 12-13.

52. Nicole Dyonnet, "Impiétés provinciales au XVIII siècle », Histoire, économie et société, IX/3 (1990), p. 407.

53. Pièce produite dans le cadre du conflit entre les consuls et le curé de Fitou (1784), citée par Blanc, art. cit., p. 884.

54. Le Rituel de Toul (1700) rappelle ainsi que la messe de paroisse est « celle qui se célèbre chaque dimanche et chaque fête [...] dans l'église de la paroisse, pour et au nom de la paroisse assemblée [...]; c'est là que se font les instructions publiques; c'est là qu'on publie ce qui est à publier, c'est là qu'on apprend des choses qu'il faut savoir, et qu'on apprend presque jamais ailleurs. »; cité par Cheyronnaud, op. cit., p. 274. 
et sarcasme (les fidèles assistent malgré tout à la messe basse du prêtre, certains rient), le plain-chant est le fait de mal élevés.

C'est paradoxalement à la précipitation des transformations de la fin du siècle que le chantre de paroisse devra sa survie provisoire. À partir de 1792, lorsque les prêtres seront chassés ou se cacheront, les maîtres d'école présideront des messes blanches, endossant pleinement à cette occasion la fonction rituelle qu'ils partageaient avec les clercs jusqu'alors ${ }^{55}$. La reconstruction concordataire confirmera même leur place: les chœurs d'église et les écoles auront besoin d'eux, immédiatement. La présence des chantres au village, encore perceptible au milieu du siècle dernier, se maintiendra pourtant dans un paysage profondément renouvelé. Animalisé par ses opposants ${ }^{56}$, progressivement coupé de sa matrice scolaire à partir de la loi Guizot (1833), environné par de nouvelles conceptions du chant grégorien en texte et en pratique ${ }^{57}$, le chantre subira désormais les attaques, bientôt victorieuses, de l'agressive beauté du chant ecclésiastique.

Xavier.Bisaro@univ-montp3.fr

55. $C f$. Xavier Bisaro, «Les maitres chanteurs du Directoire : une anthropologie sonore du culte laïcal », Revue de musicologie, XCIV/2 (2008), p. 433-450.

56. Parmi les innombrables portraits de chantres au XIX ${ }^{\mathrm{e}}$ siècle, celui de Porte (Des moyens de propager le goût de la musique en France, 1838) en est un spectaculaire exemple : «Ils ignorent complètement les régles du plain-chant et de la langue latine. Les traits de leur visage se contractent hideusement, et leurs sales mains se tordent, tandis que d'une voix glapissante ils dénaturent les airs et les paroles. Ils mettent leur amour propre à étourdir l'auditoire, ils croient chanter admirablement quand ils détonnent à tue-tête "; cité par Cheyronnaud, op. cit., p. 303.

57. À compter des débuts de la Monarchie de Juillet, le plain-chant sera au cœur de la dialectique restauratrice promue par dom Guéranger. En matière de chant ecclésiastique, l'idéal moyenâgeux de ce dernier sera bientôt galvanisé par l'encouragement de Pie IX et d'évêques à la réforme des pratiques chantées cultuelles, par la découverte et l'exploitation de manuscrits anciens et, plus généralement, par l'accession du plain-chant au rang de sujet de débat public alimenté par des publications spécialisées (ecclésiastiques et/ou scientifiques) mais aussi des organes de presse généralistes (L'Univers de Louis Veuillot notamment). Sur cette évolution, $c f$. Xavier Bisaro, « La plume ou le goupillon? - Le manuscrit H 159 de Montpellier entre érudition et restauration grégorienne ", La musique ancienne : entre historiens et musiciens, Genève, Droz, à paraître en 2010. 


\section{BIBLIOGRAPHIE DES TRAITÉS CITÉS}

CARRÉ R. (frère), Le Maistre des novices dans l'art de chanter ou Regles générales, courtes, faciles, et certaines, pour apprendre parfaitement le plein-chant, Paris, Chez Le Breton, 1744.

Cousin de Contamine, Traité critique du Plain-chant, Paris, Chez P.G. Le Mercier, 1749.

Hardouin H., Méthode nouvelle, courte et facile pour apprendre le plainchant, à l'usage du diocèse de Reims, Charleville, s. n., 1762.

ImBert, Nouvelle Méthode ou Principes raisonnés du plein-chant, dans sa perfection, tirés des éléments de la musique, Paris, Chez la Veuve Ballard \& Fils et Brocas, 1780.

LA FeILlÉE F. (de), Méthode nouvelle pour apprendre parfaitement les regles du plain-chant et de la psalmodie, Poitiers, Chez Jean Faulcon, 1748 .

LEBEUF J. (abbé), Traité historique et pratique sur le chant ecclésiastique, Paris, Cl.-J.-B. et Jean-Th. Hérissant, 1741.

Oudoux, Méthode nouvelle pour apprendre facilement le plain-chant, Paris, Chez Augustin-Martin Lottin aîné, 1772.

Poisson L., Traité théorique et pratique du plain-chant appelé Grégorien, Paris, Chez Ph. N. Lottin \& J.H. Butard, 1750.

Poisson (abbé), Nouvelle Méthode pour apprendre le plain-chant, Rouen, Labbey, 1789. 